\title{
Влияние нейтронного облучения на спектр дефектов с глубокими уровнями в GaAs, изготовленном методом жидкофазной эпитаксии в атмосфере водорода и аргона
}

\author{
(С) М.М. Соболев, Ф.Ю. Солдатенков
}

Физико-технический институт им. А.Ф. Иоффе Российской академии наук, 194021 Санкт-Петербург, Россия

E-mail: m.sobolev@mail.ioffe.ru, f.soldatenkov@mail.ioffe.ru

Поступила в Редакцию 12 августа 2021 г.

В окончательной редакции 27 августа 2021 г.

Принята к публикации 27 августа 2021 г.

Представлены результаты экспериментальных исследований методами вольт-фарадных характеристик и нестационарной спектроскопии глубоких уровней плавных высоковольтных диодов $p^{+}-p^{0}-i-n^{0}-\mathrm{GaAs}$, изготовленных методом жидкофазной эпитаксии при температурах начала кристаллизации $900^{\circ} \mathrm{C}$ из одного раствора-расплава за счет автолегирования фоновыми примесями, в атмосфере водорода или аргона, до облучения нейтронами и после. После облучения нейтронами в спектрах нестационарной спектроскопии глубоких уровней обнаружены широкие зоны кластера дефектов с акцептороподобными отрицательно заряженными ловушками в $n^{0}$-слое, возникающие в результате эмиссии электронов из состояний, расположенных выше середины запрещенной зоны. Обнаружено, что различия вольт-фарадных характеристик структур, выращенных в атмосфере водорода и аргона, обусловлены разными дозами облучения $p^{+}-p^{0}-i-n^{0}$-структур и разной степенью компенсации мелких донорных примесей глубокими ловушками в слоях.

Ключевые слова: GaAs, нейтронное облучение, емкостная спектроскопия, $p^{0}-i-n^{0}$-переход, жидкофазная эпитаксия, водород, аргон.

DOI: 10.21883/FTP.2022.01.51812.9729

\section{1. Введение}

Арсенид галлия является сегодня наиболее широко используемым полупроводником для производства широкого класса приборов СВЧ электроники и импульсной техники. Биполярные GaAs-приборы с $p-i-n$-переходами различного уровня мощности и разных рабочих напряжений нашли широкое применение в качестве смесительных, детекторных, ограничительных, генераторных и коммутационных приборов. Современная технология изготовления подобных приборов включает широкий арсенал методов радиационного регулирования характеристик полупроводниковых приборов и основана на радиационных процессах при контролируемом введении точечных дефектов в слои приборных полупроводниковых структур после полного завершения всех операций их изготовления. С другой стороны, изучение влияния радиационных воздействий на приборные структуры необходимо для выяснения области надежной работы GaAs-приборов в условиях различных видов радиации.

Существенное влияние на временны́е характеристики арсенид-галлиевых диодов (время жизни неравновесных носителей заряда и время нарастания перепадов напряжения), а также на блокируемое диодными структурами напряжение будут оказывать дефекты с глубокими уровнями (ГУ), образующиеся в процессе эпитаксиального роста структур. Ранее в работах [1-3] нами было показано, что в слоях $p^{+}-p^{0}-i-n^{0}$-диодов на основе GaAs, выращенных при температурах начала кристаллизации
$T_{b}=650-800^{\circ} \mathrm{C}$, в спектрах DLTS (deep level transient spectroscopy) обнаруживаются дырочные ловушки HL5 и HL2 [4], характерные для слоев GaAs, полученных методом жидкофазной эпитаксии (ЖФЭ) в атмосфере водорода. При повышении температуры начала кристаллизации до $T_{b} \geq 850^{\circ} \mathrm{C}$ в эпитаксиальных слоях $\mathrm{GaAs}$ помимо дефектов HL5 и HL2 наблюдалось образование дефекта, подобного EL2 [2,3]. Как было отмечено в работах $[5,6]$, имеются существенные различия процессов дефектообразования при электронном, нейтронном и протонном облучении полупроводниковых материалов. Наличие ограниченного числа ловушек в слоях $\mathrm{GaAs}$ облегчает процесс изучения радиационных дефектов, образующихся при нейтронном облучении в этих слоях. Во время столкновений твердых сфер, происходящих между нейтронами и атомами решетки, большое количество энергии может передаваться первичному атому отдачи (knock-on atom) [7]. Энергичный, отбивающийся от удара атом (recoiling knock-on atom) способен вызвать плотный каскад из нескольких сотен смещений. Этот плотный кластер дефектов захватывает подвижные носители из ближнего окружения, при этом становится заряженным и создает компенсирующую обедненную область вокруг ядра дефекта. При сравнении результатов DLTS-измерений было обнаружено, что на образцах, облученных быстрыми нейтронами [5,7], получали спектры, которые были больше похожи на спектры образцов, имплантированных тяжелыми ионами, чем образцов, подвергнутых бомбардировке протонами [5-7], несмотря 
на то что массы протонов и нейтронов практически равны. Эти различия связаны с тем, что взаимодействие нейтронов с атомами решетки происходит исключительно за счет столкновения ядер решетки и нейтроны генерируют кластеры более эффективно, чем протоны. Эффекты нейтронного повреждения в полупроводниках также заметно отличаются по сравнению с точечными дефектами и простыми дефектно-примесными комплексами. Кроме того, в работе [7] для нейтронноиндуцированной ловушки кластера повреждений было обнаружено, что она может быть одинаково эффективной ловушкой для основных носителей заряда в материалах как $n$-, так и $p$-типа проводимости, что отличает ее от обычной ловушки в объемном полупроводнике. Экспериментальные доказательства существования кластера дефектов большого размера были получены для GaAs в работе [8], где, как и в [7], обнаружили, что вид DLTS-спектра, облученного нейтронами GaAs, кардинально отличается от характерного для GaAs, облученного электронами. Электронное облучение позволяет создавать вакансии и межузельные атомы в обеих подрешетках [9]. В DLTS-спектрах после нейтронного или ионного облучения наблюдаются два широких, почти безликих, пика - один, связанный с эмиссией носителей из глубоких состояний верхней половины запрещенной зоны, обозначаемый как „U-полоса“, и другой - в нижней половине зоны, отмеченный как „L-полоса“ $[5,6,8]$. Высокотемпературный край широкой DLTS-полосы обрезался при одной и той же температуре как в $n$-, так и в p-GaAs. По мнению авторов, это означает, что обрезание происходит из-за формирования спектра DLTS, ожидаемого для непрерывной плотности дефектных состояний, охватывающей середину запрещенной зоны. Эти измерения позволили авторам сформулировать модель каскадов дефектов, которые представляют собой области суженной запрещенной зоны с неоднородно расширенными уровнями дефектов. Были проведены исследования кинетики захвата, которые показали, что кинетика захвата одинакова для $n$ - и $p$-GaAs. По мнению авторов работы [8] это связано с наличием кластеров дефектов в виде отрицательно и положительно заряженных ловушек и указывает на то, что $U$-полоса $n$-GaAs в основном состоит из акцептороподобных, а $L$-полоса $p$-GaAs - в значительной степени из донороподобных дефектов. Ранее было описано несколько моделей для широкой $U$-полосы DLTS. Первая заключается в том, что $U$-полоса состоит из неразрешенных пиков DLTS и обусловлена прямой тепловой эмиссией электронов из широкой плотности состояний, образуемой конкретными дефектами [10]. В этой модели $U$-полоса могла возникнуть либо из-за множества нижележащих дефектов с близко расположенными пиками DLTS [9], либо из-за меньшего количества основных дефектов с расширенными и смещенными пиками DLTS, вызванными сильным электрическим полем внутри кластера дефектов. Вторая модель была предложена в работе, в которой доказывается, что $U$-полоса в спектре DLTS возникает из-за непрямой многоступенчатой эмиссии электронов из центра EL2 при более низкой температуре, чем „обычный“ пик EL2, и эмиссия электронов происходит через близлежащие мелкие дефекты в кластере [6]. В работе [11] наблюдали изменения электронных свойств в облученном нейтронами GaAs, которые связывали с удалением носителей заряда на различные энергетические уровни в запрещенной зоне дефектов смещения, образующих кластер. Удаление носителя в основном связывали с захватом основных носителей этими уровнями.

Цель данной работы заключалась в выявлении влияния нейтронного облучения на образование и поведение глубоких дефектов в слоях GaAs $p^{+}-p^{0}-i-n^{0}$-диодов, изготовленных методом ЖФЭ в атмосфере водорода или аргона, с помощью методов емкостной спектроскопии: вольт-фарадных $(C-U)$ характеристик и нестационарной спектроскопии глубоких уровней (DLTS). $C-U$-измерения используются для определения профилей распределения эффективной концентрации носителей заряда в зависимости от температуры образца и оптической подсветки. DLTS-метод применяется для изучения и идентификации примесей и дефектов с глубокими уровнями в слоях $p^{+}-p^{0}-i-n^{0}$-диодов на основе GaAs.

\section{2. Образцы}

Выращивание высоковольтных $p^{0}-i-n^{0}$-структур на основе GaAs проводилось на $p^{+}$-подложках GaAs c ориентацией $(100)$, легированных цинком до $\sim 5 \cdot 10^{18} \mathrm{~cm}^{-3}$, модифицированным методом ЖФЭ из ограниченного объема раствора-расплава $\mathrm{Ga}-\mathrm{As}$ в кварцевом контейнере при температурах от $900^{\circ} \mathrm{C}$ до комнатной в атмосфере водорода или аргона (описание методики изготовления таких структур содержится в [12-15]). Распределение концентрации свободных носителей заряда по толщине плавных $p^{0}-i-n^{0}$-переходов на основе $\mathrm{GaAs}$, выращенных в атмосфере водорода и аргона, представлено на рис. 1. Профили распределения свободных носителей заряда получали при послойном стравливании структуры из вольт-фарадных характеристик обратносмещенного барьера Шоттки с помощью ртутного зонда.

Изготовление экспериментальных образцов этих структур двух типов было идентичным. На следующем этапе изготовления диодных структур на $p^{+}-p^{0}-i-n^{0}$ переходы после удаления (стравливания) $\sim 20$ мкм поверхностного слоя в графитовой поршневой кассете доращивали сильно легированные теллуром эмиттерные слои $n^{+}$-GaAs диодов. Образцы диодов (чипы) имели форму мезаструктур, которые получали путем химического стравливания активных слоев до подложки. На подложку $p^{+}$-GaAs наносили многослойный контакт $\mathrm{AgMn}-\mathrm{Ni}-\mathrm{Au}$, а на эмиттер $n^{+}$-GaAs наносили $\mathrm{AuGe}-\mathrm{Ni}-\mathrm{Au}$. 


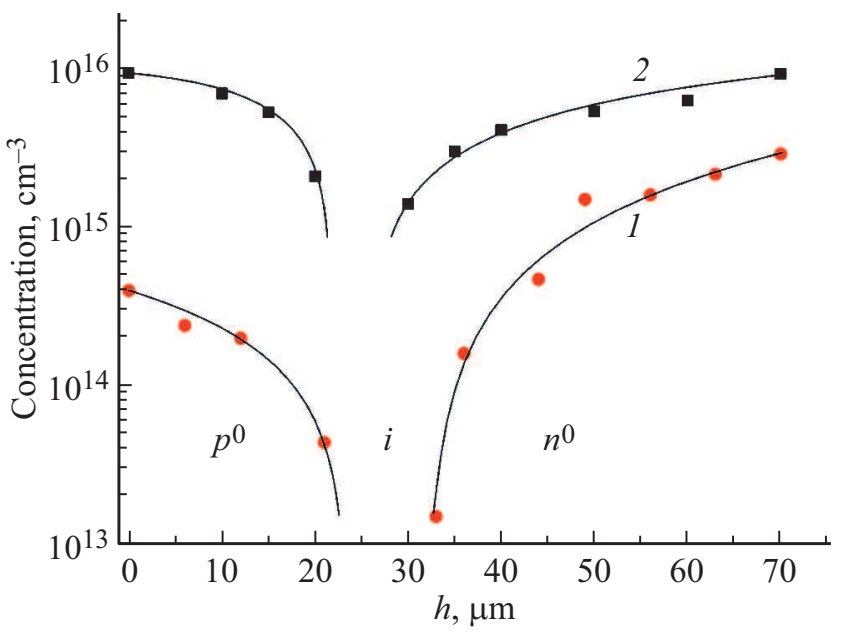

Рис. 1. Профили распределения концентрации свободных носителей заряда по толщине $h$ для образцов, выращенных в aтмосфере $\mathrm{H}_{2}$ (1) и $\mathrm{Ar}(2)$.

После изготовления чипов часть из них подверглась облучению быстрыми нейтронами с энергией $~ 1.0$ МэВ и четырьмя разными величинами потока (флюенса) $F=2.9 \cdot 10^{13}, 8.5 \cdot 10^{13}, 1.6 \cdot 10^{14}, 3.6 \cdot 10^{14} \mathrm{~cm}^{-2}$. Диоды, высоковольтные $p^{0}-i-n^{0}$-переходы которых были выращены в атмосфере водорода, оказались менее стойкими к воздействию нейтронов - такие диоды переставали пропускать электрический ток в прямом направлении после облучения нейтронами с флюенсом от $8.5 \cdot 10^{13} \mathrm{~cm}^{-2}$ и выше. Диоды с высоковольтными $p^{0}-i-n^{0}$-переходами, выращенными в атмосфере аргона, переставали пропускать электрический ток в прямом направлении после облучения нейтронами с величиной флюенса только от $3.6 \cdot 10^{14} \mathrm{~cm}^{-2}$. Поэтому для исследования влияния нейтронного облучения на образование и поведение глубоких дефектов были отобраны диоды с высоковольтным $p^{0}-i-n^{0}$-переходом, выращенном в атмосфере водорода, после облучения нейтронами с величиной флюенса $F=2.9 \cdot 10^{13} \mathrm{~cm}^{-2}$, а диоды с высоковольтным $p^{0}-i-n^{0}$-переходом, выращенном в атмосфере аргона, - после облучения нейтронами с величиной флюенса $F=1.6 \cdot 10^{14} \mathrm{~cm}^{-2}$.

\section{3. Результаты и обсуждение}

Были проведены исследования $C-U$-характеристик (рис. 2 и 3) и DLTS-спектров (рис. 4) чипов $p^{+}-p^{0}-i-n^{0}$-структур на основе эпитаксиальных слоев GaAs, выращенных методом ЖФЭ в атмосфере водорода и аргона, непосредственно с помощью DLTSспектрометра (DL4600 фирмы BIORAD, Англия) с интегратором Вoxcar. Исследования $C-U$-характеристик исходных диодов и после их облучения нейтронами проводили при различных условиях измерения: в темноте и при воздействии белым светом в процессе измерения $C-U$-характеристик. Для удобства представления результатов $C-U$-измерений и их дальнейшей интерпретации так же, как и в $[1,16-21]$, использовался метод дифференциальной емкости, из $C-U$-характеристик рассчитывались профили распределения эффективной концентрации свободных носителей заряда $\left(n^{*}\right)$ по толщине $(W)$ слоя объемного заряда (CO3). Ранее мы уже исследовали подобные $p^{+}-p^{0}-i-n^{0}$-структуры на основе эпитаксиального GaAs, выращенные в атмосфере водорода $[1,16,17]$, в которых наблюдаемые изменения $C-U$-характеристик (рис. 2,a) были связаны с температурным ходом диффузионного потенциала. Оптическая перезарядка глубоких ловушек акцепторного типа в $i$-слое и примыкающих к нему $p^{0}$ и $n^{0}$-слоях не приводила к существенному изменению эффективной концентрации носителей в зоне проводимости эпитаксиальных слоев $\left(n^{*}\right)$. Толщина слоя объемного заряда $(W)$ при температуре измерения $300 \mathrm{~K}$ составила величину $\sim 6.5$ мкм, при этом эффективная концентрация носи-
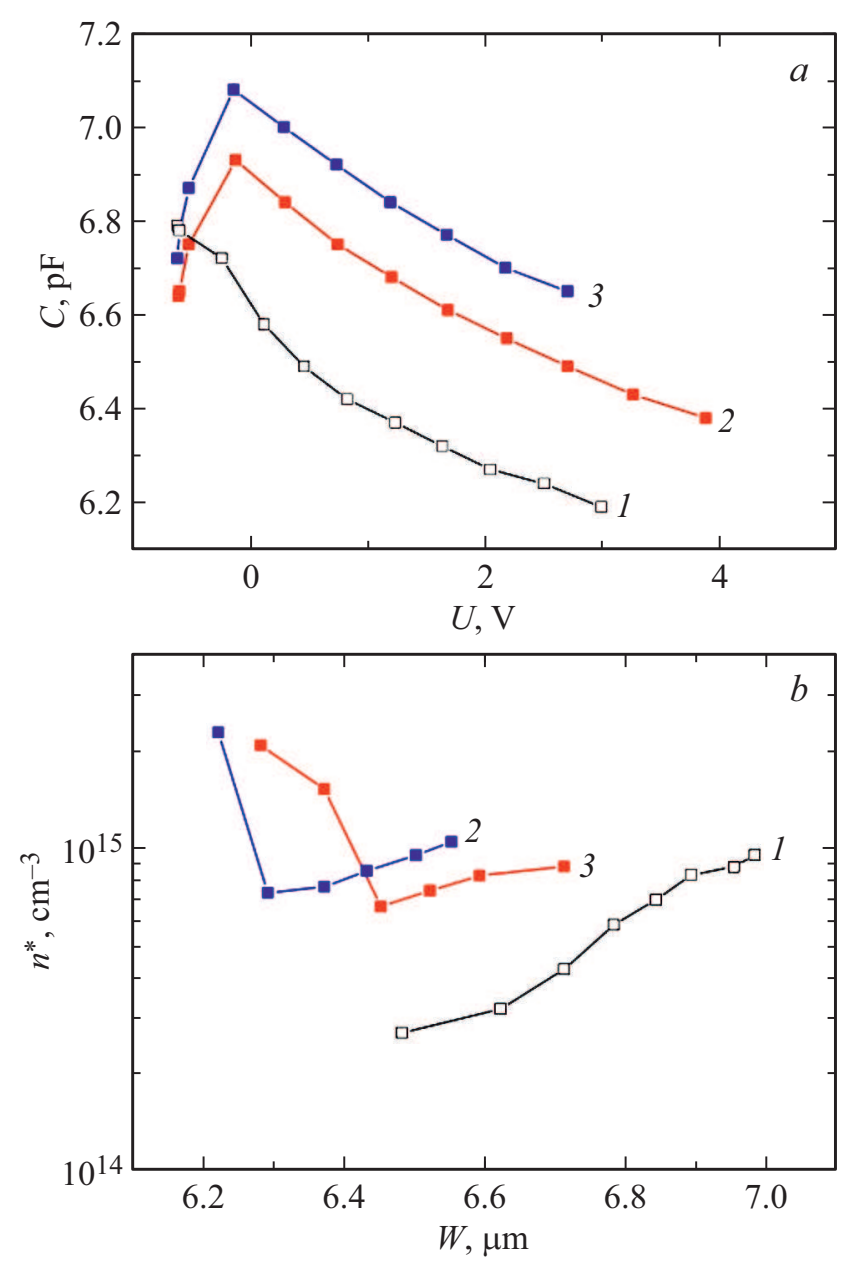

Рис. 2. $C-U$-характеристики $(a)$ и профили распределения эффективной концентрации свободных носителей заряда $n^{*}$ по толщине $W$ слоя объемного заряда $(b)$ диодов $p^{+}-p^{0}-i-n^{0}-\mathrm{GaAs}$, выращенных в атмосфере водорода: исходных $(1)$, облученных нейтронами $(2,3)$, в темноте $(1,2)$ и при оптической подсветке (3). 

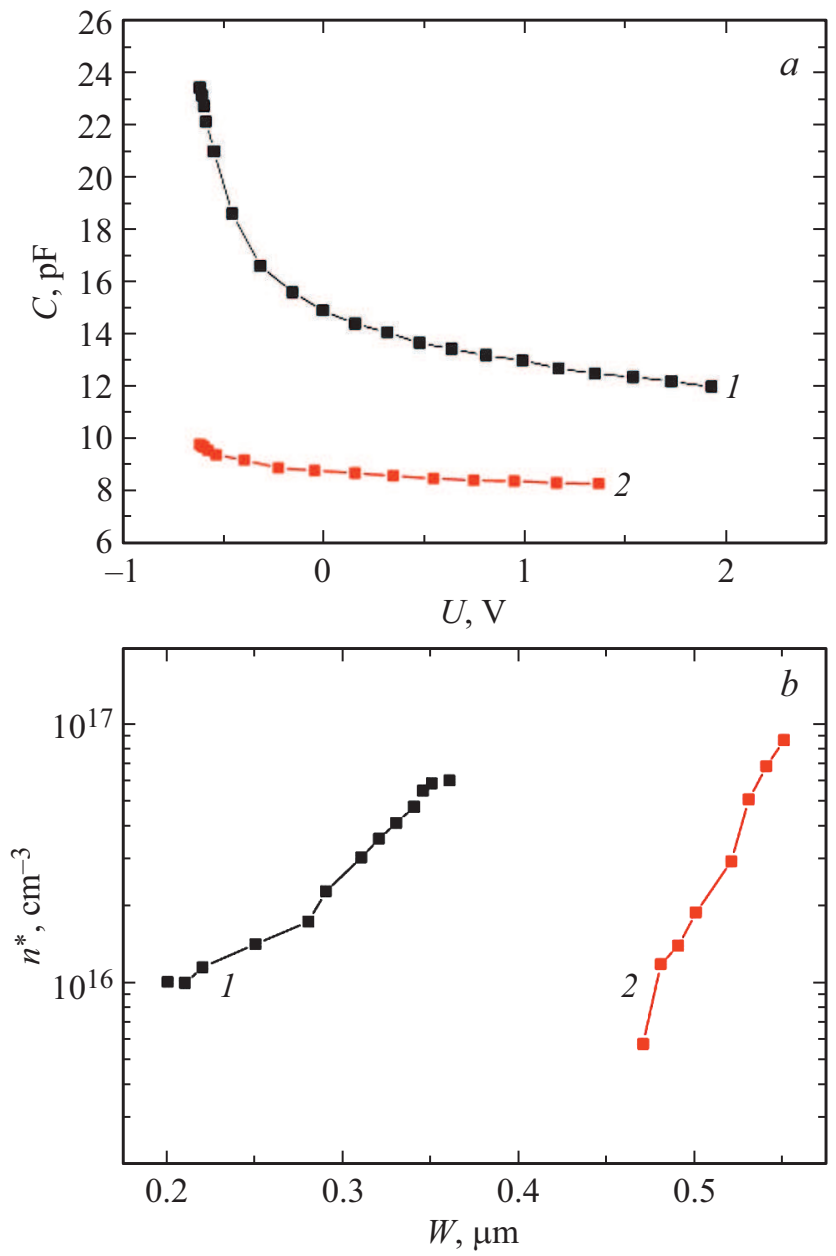

Рис. 3. $C-U$-характеристики $(a)$ и профили распределения эффективной концентрации свободных носителей заряда $n^{*}$ по толщине $W$ слоя объемного заряда $(b)$ диодов $p^{+}-p^{0}-i-n^{0}-\mathrm{GaAs}$, выращенных в атмосфере аргона: исходных (1), облученных нейтронами (2). Измерения проводились в темноте.

телей была $n^{*} \approx(2.7-9.7) \cdot 10^{14} \mathrm{~cm}^{-3}$ (рис. $\left.2, b\right)$. Как было показано ранее $[1,16,17], p^{0}$-, $i$ - и $n^{0}$-слои были сформированы за счет автолегирования эпитаксиальных слоев мелкими и глубокими примесями и дефектами акцепторного типа, в качестве которых выступали центры HL5 и HL2. После облучения нейтронами $p^{+}-p^{0}-i-n^{0}$-структуры, выращенной в атмосфере водорода, произошли кардинальные изменения $C-U$-характеристики (рис. 2,a). В отличие от $C-U$-характеристики исходной структуры, профиль $C-U$-характеристики структуры, облученной нейтронами, имел пик с максимумом в области прямого напряжения смещения $(U=0.14 \mathrm{~B})$. При дальнейшем увеличении прямого напряжения емкость резко падала. С ростом обратного напряжения емкость падает в соответствии с изменением диффузионной емкости, и $C-U$-характеристика имеет вид, типичный для $p-n$-перехода с глубокими уровнями. Образование пика, во-первых, связывается с существованием электрически активных глубоких ловушек в области $p-n$-перехода [22,23]. Во-вторых, образование пика $C-U$-характеристики при прямом смещении может быть связано с протеканием тока, ограниченного пространственным зарядом (SCL), состоящего из суммы дрейфового и диффузионного токов $[23,24]$. При прямом смещении с увеличением диффузии инжектированных носителей емкость растет. При достижении режима SCL, когда начинает доминировать дрейф носителей, емкость проходит через пик и падает. Следующее отличие, которое произошло после облучения структуры нейтронами, - емкость в базовой области $p-i-n$-структуры увеличилась по сравнению с необлученной структурой, так как произошел рост эффективной концентрации носителей в зоне проводимости эпитаксиальных слоев $n^{*}$ и, следовательно, уменьшилась толщина слоя объемного заряда. Как было установлено ранее [2,3], формирование $p^{0}$-, $i$ - и $n^{0}$-слоев происходит за счет компенсации мелких фоновых примесей глубокими донорными (EL2) и акцепторными (HL2, HL5, Ga $\mathrm{As}$ ) дефектами. Концентрация акцепторных уровней HL2 и HL5 в $i$-слое оказывается сравнимой с концентрацией мелких доноров. При облучении нейтронами происходят, повидимому, два события: во-первых, уменьшается концентрация уровней HL2 и HL5 в $i$-слое; во-вторых, образуются кластеры разрушения, проявляющие свойства ловушек акцепторного типа $[7,11]$, причем концентрация этих ловушек меньше, чем концентрация уровней HL2 и HL5. Также для $p^{+}-p^{0}-i-n^{0}$-структуры была выявлена зависимость $C-U$-характеристик от наличия оптической подсветки при измерении образца (рис. $2, a, b$ ). Подсветка структур приводила к уменьшению толщины СО3 при $300 \mathrm{~K}$. Наиболее вероятной причиной таких изменений толщины СО3 от подсветки может быть образование пространственно-локализованных глубоких состояний донорного и акцепторного типов на границах $p^{+}-p^{0}-i-n^{0}$-структуры и в $i$-слое, обусловленных кластерами разрушения. Влияние плотности локализованных состояний на $C-U$-характеристику $p-n$-перехода исследовалось подробно в работах [25-27]. Для $C-U$-характеристик и профилей распределения $n^{*}(W)$ $p^{+}-p^{0}-i-n^{0}$-структур на основе эпитаксиальных слоев GaAs, изготовленных методом ЖФЭ в атмосфере аргона (рис. $3, a, b)$, выявились существенные отличия от аналогичных структур, полученных в атмосфере водорода (рис. 2, $a, b$ ). Во-первых, значения емкости слоев оказались в $\sim 2-3$ раза выше и, соответственно, при этом эффективная концентрация носителей $n^{*}$ была $\approx(0.95-5.9) \cdot 10^{16} \mathrm{~cm}^{-3}$, толщина слоя объемного заряда $(W)$ при температуре измерения $300 \mathrm{~K}$ составила всего 0.2 мкм. Получение $p^{+}-p^{0}-i-n^{0}$-структур методом ЖФЭ в атмосфере инертного аргона в отличие от структур, полученных в атмосфере водорода, повидимому, не способствует эффективной очистке эпитаксиальных слоев от фоновых примесей в процессе роста. После облучения нейтронами $p^{+}-p^{0}-i-n^{0}$-структуры, выращенной в атмосфере аргона, не произошло суще- 
ственных изменений $C-U$-характеристики (рис. $3, a$ ), если не считать уменьшения емкости слоев в $\sim 2$ раза и смещения профиля эффективной концентрации носителей $n^{*}$ на 0.27 мкм (рис. $\left.3, b\right)$.

Итак, изменения $C-U$-профилей для структур $p^{+}-p^{0}-i-n^{0}$-GaAs, изготовленных в атмосфере водорода и аргона, различаются с точностью до наоборот: в первом случае после облучения структуры нейтронами емкость увеличивалась, а во втором - уменьшалась. Различия, по-видимому, обусловлены разными дозами облучения структур $p^{+}-p^{0}-i-n^{0}$-GaAs: первая структура облучалась нейтронами с флюенсом $2.9 \cdot 10^{13} \mathrm{~cm}^{-2}$, и в ней, как было объяснено, компенсация мелких доноров в $i$-слое глубокими акцепторными ловушками уменьшилась, а емкость соответственно увеличилась. Структура, выращенная в атмосфере аргона, облучалась нейтронами с флюенсом $1.6 \cdot 10^{14} \mathrm{~cm}^{-2}$, что приводило к увеличению концентрации кластеров разрушения приблизительно на порядок, и степень компенсации донорных примесей глубокими ловушками значительно возрастала, эффективная концентрация носителей $n^{*}$ уменьшалась, а толщина слоя объемного заряда увеличивалась. Оптическая перезарядка глубоких ловушек акцепторного типа в $i$-слое и примыкающих к нему $p^{0}$-, $n^{0}$-слоях не приводила к изменению эффективной концентрации носителей в зоне проводимости эпитаксиальных слоев $\left(n^{*}\right)$ как до, так и после облучения нейтронами.

Для диодов $p^{+}-p^{0}-i-n^{0}-\mathrm{GaAs}$, изготовленных методом ЖФЭ в атмосфере водорода (рис. 4, $a, b)$ и аргона (рис. 4,c), были измерены DLTS-спектры при различных значениях напряжений смещения $U_{r}$ и импульсов заполнения $U_{f}$, до облучения нейтронами и после. Измерения DLTS-спектров проводились в темноте и при воздействии белым светом. На рис. 4 показаны DLTS-спектры структур $p^{+}-p^{0}-i-n^{0}$-GaAs при напряжении смещения $U_{r}=-1.0 \mathrm{~B}$ и для двух значений импульса заполнения $U_{f}=0.01$ В (спектры 1 и 3) и 0.5 В (спектры 2 и 4), исходной структуры (1 и 2) и структуры после облучения (3 и 4). Спектр 4 был измерен при воздействии белым светом. Ранее $[1,16,17]$ мы уже исследовали DLTSспектры структур $p^{+}-p^{0}-i-n^{0}$-GaAs, выращенных в атмосфере водорода, которые представляли собой плавный $p^{0}-i-n^{0}$-переход с концентрацией свободных носителей заряда в $i$-слое $\sim\left(10^{13}-10^{14}\right) \mathrm{cm}^{-3}$. В DLTS-спектрах этих структур, измеренных при положительном и отрицательном значениях импульса заполнения $U_{f}$, удалось выявить глубокие ловушки неосновных и основных носителей в $n^{0}$ - и $p^{0}$-слоях, которые были идентифицированы как известные ловушки, характерные для GaAs. При измерении DLTS-спектров в темноте при положительном значении импульса заполнения $U_{f}=0.01 \mathrm{~B}$ и $U_{r}=-1.0 \mathrm{~B}$ наблюдалось два положительных DLTSпика, Н4 и Н5, появление которых связывалось с захватом и эмиссией дырок на акцепторные ловушки неосновных носителей в $n^{0}$-слое (см. рис. 4, $a, b$; отметим, что на рис. 4, $b$ показаны спектры 1,3 и 4 рис. $4, a$ в увеличенном масштабе). Энергия термической
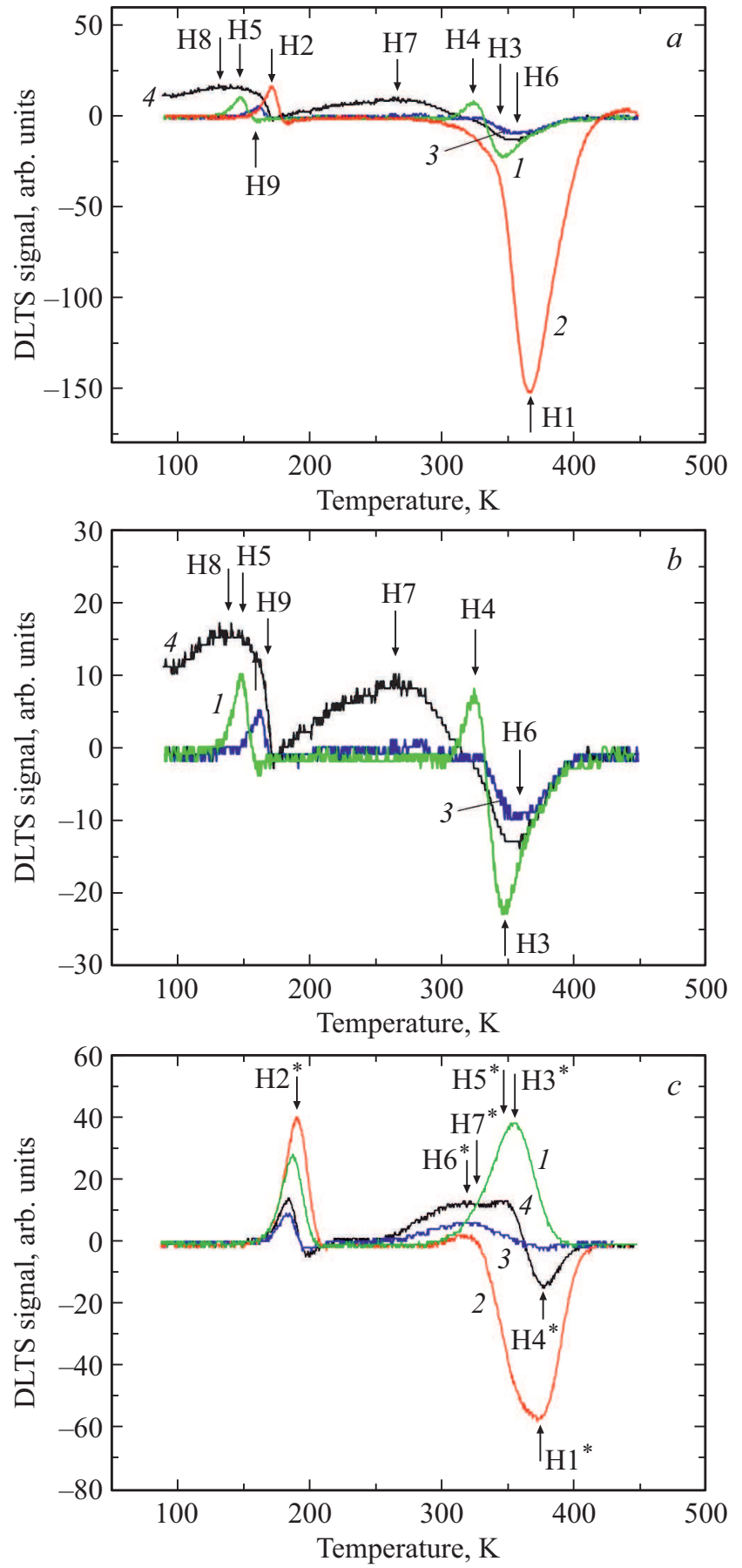

Рис. 4. DLTS-спектры диодов $p^{+}-p^{0}-i-n^{0}$-GaAs, выращенных в атмосфере водорода $(a, b)$ и аргона $(c)$, измеренные при напряжении смещения $U_{r}=-1.0 \mathrm{~B}$ и напряжениях импульса заполнения $U_{f}=0.01$ (спектры 1 и 3 ) и $0.5 \mathrm{~B}$ (спектры 2 и 4 ), в темноте $(1,2,3)$ и при воздействии белым светом (4), исходных $(1,2)$ и облученных нейтронами $(3,4)$. Темп окон $200^{-1}$ с. (Цветной вариант рисунка представлен в электронной версии статьи).

активации и сечение захвата одной из ловушек с ГУ, а именно Н5, определенные с помощью зависимости Аррениуса, были равны соответственно: $E_{t}=286$ мэВ, 
Идентификация электронных и дырочных ловушек в структурах $p^{+}-p^{0}-i-n^{0}$-GaAs, выращенных методом ЖФЭ в атмосфере водорода и аргона, до и после нейтронного облучения

\begin{tabular}{|c|c|c|c|c|c|}
\hline Глубокий уровень & Энергия, мэВ & Сечение захвата, см² & Концентрация ГУ, см$^{-3}$ & Идентификация & Ссылки \\
\hline \multicolumn{6}{|c|}{ GaAs, выращенный в атмосфере водорода, до облучения } \\
\hline $\begin{array}{l}\mathrm{H} 1 \\
\mathrm{H} 2 \\
\mathrm{H} 3 \\
\mathrm{H} 5\end{array}$ & $\begin{array}{l}649 \\
329 \\
520 \\
286 \\
\end{array}$ & $\begin{array}{l}6.44 \cdot 10^{-16} \\
1.61 \cdot 10^{-14} \\
3.25 \cdot 10^{-17} \\
2.74 \cdot 10^{-14}\end{array}$ & $\begin{array}{l}6.36 \cdot 10^{14} \\
4.75 \cdot 10^{13} \\
6.58 \cdot 10^{13} \\
2.71 \cdot 10^{14} \\
\end{array}$ & $\begin{array}{c}\text { HL2 }\left[\mathrm{V}_{\mathrm{Ga}}+\right] \\
\text { HL6, HL5 } \\
\text { HL3 [Fe] } \\
\text { H1, HB6 }\end{array}$ & $\begin{array}{l}4] \\
{[4]} \\
{[4]} \\
{[4]}\end{array}$ \\
\hline \multicolumn{6}{|c|}{ GaAs, выращенный в атмосфере водорода, после облучения нейтронами } \\
\hline $\begin{array}{l}\mathrm{H} 6 \\
\mathrm{H} 7 \\
\mathrm{H} 8 \\
\mathrm{H} 9 \\
\end{array}$ & $\begin{array}{l}727 \\
362 \\
264 \\
267 \\
\end{array}$ & $\begin{array}{l}1.49 \cdot 10^{-14} \\
3.51 \cdot 10^{-17} \\
3.09 \cdot 10^{-14} \\
9.96 \cdot 10^{-16}\end{array}$ & $\begin{array}{r}2.5 \cdot 10^{13} \\
3.10 \cdot 10^{13} \\
3.30 \cdot 10^{12} \\
2.80 \cdot 10^{12} \\
\end{array}$ & $\begin{array}{c}\mathrm{HL} 2\left[\mathrm{~V}_{\mathrm{Ga}}+\right] \\
U \text {-band } \\
\mathrm{H} 1 \\
\mathrm{H} 1, \mathrm{HB} 6 \\
\end{array}$ & $\begin{array}{c}4] \\
{[4]} \\
{[28]} \\
{[28,4]} \\
\end{array}$ \\
\hline \multicolumn{6}{|c|}{ GaAs, выращенный в атмосфере аргона, до облучения } \\
\hline $\begin{array}{l}\mathrm{H} 1^{*} \\
\mathrm{H} 2^{*} \\
\mathrm{H} 3^{*}\end{array}$ & $\begin{array}{l}699 \\
368 \\
678 \\
\end{array}$ & $\begin{array}{l}1.84 \cdot 10^{-15} \\
2.57 \cdot 10^{-14} \\
3.91 \cdot 10^{-15} \\
\end{array}$ & $\begin{array}{r}.65 \cdot 10^{15} \\
2.4 \cdot 10^{15} \\
3.60 \cdot 10^{15} \\
\end{array}$ & $\begin{array}{c}\text { HL2, HS2 } \\
\text { HL5 }\left[\mathrm{V}_{\mathrm{Ga}}+?\right] \\
\text { HL2 }\left[\mathrm{V}_{\mathrm{Ga}}+?\right]\end{array}$ & $\begin{array}{l}4] \\
{[4]} \\
{[4]} \\
\end{array}$ \\
\hline \multicolumn{6}{|c|}{ GaAs, выращенный в атмосфере аргона, после облучения нейтронами } \\
\hline $\begin{array}{l}\mathrm{H} 4^{*} \\
\mathrm{H} 5^{*} \\
\mathrm{H} 6^{*} \\
\mathrm{H} 7^{*}\end{array}$ & $\begin{array}{l}593 \\
343 \\
384 \\
685\end{array}$ & $\begin{array}{l}6.11 \cdot 10^{-17} \\
2.32 \cdot 10^{-20} \\
1.32 \cdot 10^{-18} \\
8.19 \cdot 10^{-14}\end{array}$ & $\begin{array}{l}2.96 \cdot 10^{15} \\
2.63 \cdot 10^{15} \\
2.21 \cdot 10^{15} \\
1.18 \cdot 10^{15}\end{array}$ & $\begin{array}{c}\text { HL2, HS2 } \\
\text { HS3 } \\
\text { HL3 [Fe] }\end{array}$ & $\begin{array}{l}{[4]} \\
{[4]} \\
{[4]}\end{array}$ \\
\hline
\end{tabular}

$\sigma_{p}=1.64 \cdot 10^{-14} \mathrm{~cm}^{2}$ (см. таблицу). Эта дырочная ловушка близка по параметрам к НB6 [4]. Для пика H4 не удалось определить параметры из зависимости Аррениуса. Кроме пиков ловушек неосновных носителей в DLTS-спектрах проявился пик Н3 с отрицательным знаком, который, как будет показано далее, приписывался примеси $\mathrm{Fe}$ (HL3) [4] и был ловушкой основных носителей в $p^{0}$-слое. При измерении DLTS-спектра с $U_{f}=0.5 \mathrm{~B}$ и $U_{r}=-1.0 \mathrm{~B}$ вместо него появлялся DLTSпик Н1. Он был по амплитуде существенно выше, чем пик Н3, смещался в сторону более высоких температур (рис. 4, $a, b$ ), идентифицировался как дефект HL2 [4] и не исчезал при положительном значении напряжения смещения $U_{r}=0.14 \mathrm{~B}$. Этот DLTS-спектр не показан на рис. $4, a, b$. Пик Н1 практически исчезал при $U_{f}=0.01 \mathrm{~B}$ и $U_{r}=-1.0 \mathrm{~B}$, когда импульс заполнения практически принимал нулевое значение. Для ловушки основных носителей в $n^{0}$-слое при $U_{r}=-1.0$ В такого не должно быть. Если в $n^{0}$-слое есть ловушка основных носителей, то при DLTS-измерениях пик с отрицательным знаком должен проявляться. Так как $p^{0}$-слой при этих условиях измерений не участвует в процессе зарядки и эмиссии носителей, то и никакого DLTS-пика, связанного с этим слоем не должно обнаруживаться. Поскольку импульсы заполнения и напряжения, при которых измерялись DLTS-спектры, приложены в прямом направлении $\left(U_{f}=0.5 \mathrm{~B}\right.$ и $\left.U_{r}=0.14 \mathrm{~B}\right)$, заполнение ловушек основными носителями и их эмиссия должны происходить из $p^{0}$-слоя и определяться глубокими уровнями акцепторов в $p^{0}$-слое $[16,17]$. С этими акцепторными ГУ как раз и связаны пики Н1 и Н3 (см. таблицу), принадлежащие дефектам HL2 и HL3 соответственно.

После облучения нейтронами структуры $p^{+}-p^{0}-i-n^{0}-\mathrm{GaAs}$, выращенной в атмосфере водорода, в DLTS-спектрах произошли изменения. Во-первых, произошло существенное уменьшение амплитуды пика Н1 с отрицательным знаком при $U_{f}=0.5 \mathrm{~B}$ и $U_{r}=-1.0 \mathrm{~B}$ (рис. $4, a$ ). На возможность уменьшения концентрации сигналов, связанных с дефектами HL2 и HL5, было обращено внимание в работах [7,11]. Во-вторых, при DLTS-измерениях с этими значениями $U_{f}$ и $U_{r}$ в спектре обнаружились две широкие зоны, связанные с эмиссией носителей из $n^{0}$-слоя, характерные для облученного нейтронами GaAs [5-8]. Эти полосы связывают с кластерами повреждений. В работе [8] было показано, что каскады дефектов повреждений окружены большими кулоновскими барьерами, которые препятствуют полному заполнению ловушек в поврежденных областях. Кулоновские барьеры существуют как для $n$-, так и для $p$-GaAs и указывают на то, что отрицательный заряд должен накапливаться в $n$-GaAs, а положительный - в $p$-GaAs, т. е. характер дефектной полосы состояний выше середины запрещенной зоны будет акцептороподобным, а ниже - донороподобным [8]. Принимая во внимание выводы статьи [8], делаем заключение, что, по-видимому, мы наблюдаем две широкие $U$-полосы в DLTS-спектре (рис. 4, $a, b$ ), связанные с эмиссией электронов из акцептороподобных дефектных полос, расположенных 
выше середины запрещенной зоны. DLTS-сигнал в таком случае будет иметь положительный знак, как это ранее наблюдалось для локализованных состояний дислокаций и квантовых точек $[16,17,19,29]$. Первая зона с пиком Н8 образовалась на месте низкотемпературного пика Н5, вторая, высокотемпературная зона, обозначенная как $\mathrm{H7}$, до облучения никак в DLTS-спектре не проявлялась.

Для диодов $p^{+}-p^{0}-i-n^{0}-\mathrm{GaAs}$, выращенных методом ЖФЭ в атмосфере аргона, после облучения нейтронами эффективная концентрация носителей $n^{*}$ возросла до $\sim(9.5-59) \cdot 10^{15} \mathrm{~cm}^{-3}$, что обусловливало определенные изменения в DLTS-спектрах по сравнению с необлученной структурой. Пики Н3* и Н2*, отчетливо прописанные в DLTS-спектрах при $U_{f}=-0.02 \mathrm{~B}$ и $U_{r}=-1.0 \mathrm{~B}$ и являющиеся ловушками неосновных носителей в $n^{0}$-слое исследуемых структур (рис. 4,c), позволили определить параметры из зависимости Аррениуса (см. таблицу). Они оказались связанными с дефектами HL2 и HL5. При измерении DLTSспектра исходной $p^{+}-p^{0}-i-n^{0}$-структуры с $U_{f}=0.5 \mathrm{~B}$ и $U_{r}=-1.0 \mathrm{~B}$, как и в случае структуры, выращенной в водороде, наблюдался отрицательный широкий пик $\mathrm{H} 1^{*}$, который был идентифицирован как дефект HL2. После облучения структуры $p^{+}-p^{0}-i-n^{0}$-GaAs, изготовленной в атмосфере аргона, произошли изменения в DLTS-спектрах: появилась одна широкая высокотемпературная зона, модулированная двумя пиками $\mathrm{H}^{*}$ и $\mathrm{H6}^{*}$, параметры дефектов были соответственно: $E_{t}=343$ мэВ,$\sigma_{p}=2.32 \cdot 10^{-20} \mathrm{~cm}^{2}$ и $E_{t}=384$ мэВ, $\sigma_{p}=1.32 \cdot 10^{-18} \mathrm{~cm}^{2}$. В работе [8] было установлено, что в облученном нейтронами GaAs образуется непрерывное распределение состояний дефектов, подобное тому, что имеется в аморфном кремнии. Возможно, что в облученном нейтронами GaAs реализуется механизм эмиссии носителей, который наблюдается в аморфных материалах. Низкие сечения захвата носителей характерны для аморфных материалов с плотноупакованными слоями, в которых эмиссия с глубоких состояний осуществляется посредством прыжковой проводимости через промежуточные состояния $[6,18,19]$.

\section{4. Заключение}

Проведены исследования $C-U$-характеристик и спектров DLTS высоковольтных $p^{+}-p^{0}-i-n^{0}$-диодов на основе GaAs, изготовленных методом ЖФЭ при температурах начала кристаллизации $900^{\circ} \mathrm{C}$ из одного растворарасплава за счет автолегирования фоновыми примесями в атмосфере водорода и аргона, до облучения нейтронами и после. Установлено, что обе исследуемые структуры до облучения представляли собой плавный $p^{0}-i-n^{0}$-переход. Это позволяло проводить исследования DLTS-спектров в $p^{0}$ - и $n^{0}$-слоях $p^{0}-i-n^{0}$-структур при двух различных значениях $U_{r}$, с прямым и нулевым напряжением смещения. Эти измерения способствовали выявлению дефектов с ГУ HL2 и HL5 в эпитаксиальных $p_{0^{-}}$и $n_{0}$-слоях $\mathrm{GaAs}$, выращенных в атмосфере водорода и аргона. В первой структуре их концентрации были $10^{14} \mathrm{~cm}^{-3}$, во второй $\sim 10^{15} \mathrm{~cm}^{-3}$, при этом концентрации нескомпенсированных примесей в $p^{0}$ - и $n^{0}$-слоях изменялись в диапазоне $10^{13}-10^{14} \mathrm{~cm}^{-3}$ в первой структуре, а во второй $-10^{14}-10^{16} \mathrm{~cm}^{-3}$. После облучения обеих структур $p^{0}-i-n^{0}$-GaAs нейтронами произошли существенные изменения. B GaAsструктуре, выращенной в атмосфере водорода, в $n^{0}$-слое увеличилась эффективная концентрация носителей в зоне проводимости $\left(n^{*}\right)$. Профиль $C-U$-характеристики имел пик с максимум в области прямого напряжения смещения $(U=0.14 \mathrm{~B})$. Его наличие связывается с существованием электрически активных глубоких ловушек в области $p-n$-перехода и с протеканием тока, ограниченного пространственным зарядом (SCL), состоящего из суммы дрейфового и диффузионного токов. Оптическая подсветка также приводила к изменениям профиля $C-U$-характеристики - емкость уменьшалась. Для $C-U$-характеристик и профилей распределения $n^{*}(W) p^{0}-i-n^{0}$-структур на основе эпитаксиальных слоев GaAs, изготовленных в атмосфере аргона, после облучения нейтронами выявились существенные отличия от GaAs-структур, полученных в атмосфере водорода. После облучения структуры, изготовленной в атмосфере аргона, профиль $C-U$-характеристики не изменился, однако емкости слоев уменьшились в $\sim 2$ раза. Повидимому, различия $C-U$-характеристик структур, выращенных в атмосфере водорода и аргона, обусловлены разными дозами облучения структур $p^{+}-p^{0}-i-n^{0}-\mathrm{GaAs}$ первая структура облучалась нейтронами с флюенсом $2.9 \cdot 10^{13} \mathrm{~cm}^{-2}$, и в ней компенсация мелких доноров в $i$-слое глубокими акцепторными ловушками уменьшилась, а емкость соответственно увеличилась; вторая структура облучалась нейтронами с флюенсом $1.6 \cdot 10^{14} \mathrm{~cm}^{-2}$, что привело к увеличению концентрации кластеров разрушения приблизительно на порядок, и степень компенсации донорных примесей глубокими ловушками значительно возросла. После облучения нейтронами в DLTS-спектрах обеих структур $p^{0}-i-n^{0}$-GaAs были обнаружены широкие зоны, связанные с эмиссией электронов из состояний, расположенных выше середины запрещенной зоны, кластера дефектов с акцептороподобными отрицательно заряженными ловушками $n^{0}$-слоя.

\section{Конфликт интересов}

Авторы заявляют, что у них нет конфликта интересов.

\section{Список литературы}

[1] М.М. Соболев, Ф.Ю. Солдатенков, В.А. Козлов. ФТП, 50 (7), 941 (2016).

[2] М.М. Соболев, П.Н. Брунков, С.Г. Конников, М.Н. Степанова, В.Г. Никитин, В.П. Улин, А.Ш. Долбая, Т.Д. Камушадзе, Р.М. Майсурадзе. ФТП, 25 (6), 1058 (1989). 
[3] П.Н. Брунков, С. Гайбуллаев, С.Г. Конников, В.Г. Никитин, М.И. Папенцев, М.М. Соболев. ФТП, 25 (2), 338 (1991).

[4] G.M. Martin, A. Mitonneau, A. Mircea. Electron. Lett., 13 (22), 666 (1977). http://dx.doi.org/10.1049/el:19770473

[5] G. Guiloiot. Rev. Phys. Appl., 23, 833 (1988). https://doi.org/10.1051/rphysap:01988002305083300

[6] Sh. Makram-Ebeid, P. Boher. Rev. Phys. Appl., 23, 847 (1988).

[7] C.E. Barnes, T.E. Zipperian, L.R. Dawson. J. Electron. Mater., 14 (2), 95, (1985). https://doi.org/10.1007/BF02656670

[8] R.M. Fleming, D.V. Lang, C.H. Seager, E. Bielejec, G.A. Patrizi, J.M. Campbell. J. Appl. Phys., 107, 123710 (2010). DOI: $10.1063 / 1.3448118$

[9] D. Pons, J.C. Bourgoin. J. Phys. C: Solid State Phys., 18, 3839 (1985).

[10] В.Н. Брудный, В.В. Пешев. ФТП, 37 (2), 151 (2003).

[11] J.G. Williams, J.U. Patel, A.M. Ougouag, S.-Y. Yang. J. Appl. Phys., 70, 4931 (1991). https://doi.org/10.1063/1.349039

[12] Ф.Ю. Солдатенков, В.Г. Данильченко, В.И. Корольков. ФТП, 41 (2), 217 (2007).

[13] В.Г. Данильченко, В.И. Корольков, Ф.Ю. Солдатенков. ФТП, 43 (8), 1093 (2009).

[14] V.A. Kozlov, F.Y. Soldatenkov, V.G. Danil'chenko, V.I. Korol'kov, I.L. Shul'pina. Proc. 25th Adv. Semiconductor Manufacturing Conf. (Saratoga Springs, USA, 2014) p. 139. DOI: 10.1109/ASMC.2014.6847011

[15] Л.С. Берман, В.Г. Данильченко, В.И. Корольков, Ф.Ю. Солдатенков. ФТП, 34 (5), 558 (2000).

[16] M.M. Sobolev, F.Y. Soldatenkov, L. Shul'pina. J. Appl. Phys., 123, 161588 (2018). DOI: 10.1063/1.5011297

[17] М.М. Соболев, Ф.Ю. Солдатенков. ФТП, $54(10), 177$ (2018).

[18] М.М. Соболев, О.С. Кен, О.М. Сресели, Д.А. Явсин, С.А. Гуревич. Письма ЖТФ, 44 (7), 30 (2018).

[19] M.M. Sobolev, O.S. Ken, O.M. Sreseli, D.A. Yavsin, S.A. Gurevich. Semicond. Sci. Technol., 34, 085003 (2019). https://doi.org/10.1088/1361-6641/ab2c21

[20] М.М. Соболев, Ф.Ю. Солдатенков. ФТП, 52 (2), 1072 (2020).

[21] M.M. Sobolev, F.Y. Soldatenkov, V.G. Danil'chenko. J. Appl. Phys., 128, 095705 (2020). DOI: 10.1063/5.0018317

[22] A. Sharma, P. Kumar, B. Singh, S.R. Chaudhuri, S. Ghosh. Appl. Phys. Lett., 99, 023301 (2011). https://doi.org/10.1063/1.3607955

[23] М.М. Соболев, Д.А. Явсин, С.А. Гуревич. ФТП, 53 (10), 1431 (2019).

[24] H. Silva, H.L. Gomes, Yu.G. Pogorelov, P. Stallinga, D.M. de Leeuw, J.P. Araujo, J.B. Sousa, S.C.J. Meskers, G. Kakazei, S. Cardoso, P.P. Freitas. Appl. Phys. Lett., 94, 202107 (2009). DOI: 10.1063/1.3134484

[25] E.S. Yang. J. Appl. Phys., 45 (9), 3801 (1974).

[26] М.М. Соболев, А.В. Гитцович, М.И. Папенцев, И.В. Кочнев, Б.С. Явич. ФТП, 26 (10), 1760 (1992).

[27] Д.В. Давыдов, А.Л. Закгейм, Ф.М. Снегов, М.М. Соболев, А.Е. Черняков, А.С. Усиков, Н.М. Шмидт. Письма ЖТФ, 33 (4), 11 (2007).

[28] D. Stievenard, X. Boddaert, J.C. Bourgoin. Phys. Rev. B, 34, 4048 (1986). https://doi.org/10.1103/PhysRevB.34.4048

[29] M.M. Sobolev, A.R. Kovsh, V.V. Ustinov, A.Y. Egorov, A.E. Zhukov, Y.G. Musikhin. J. Electron. Mater., 28, 491 (1999).

Редактор Л.В. Шаронова

\section{Effect of neutron irradiation \\ on the spectrum of deep-level defects in GaAs grown by liquid-phase epitaxy in hydrogen or argon ambient}

\author{
M.M. Sobolev, F.Yu. Soldatenkov \\ loffe Institute, \\ 194021 St. Petersburg, Russia
}

\begin{abstract}
The results of experimental studies of capacitancevoltage characteristics, spectra of deep-level transient spectroscopy of graded high-voltage $\mathrm{GaAs} p^{+}-p^{0}-i-n^{0}$ diodes fabricated by liquid-phase epitaxy at a crystallization temperature of $900^{\circ} \mathrm{C}$ from one solution-melt due to autodoping with background impurities, in a hydrogen or argon ambient, before and after irradiation with neutrons. After neutron irradiation, deep-level transient spectroscopy spectra revealed wide zones of defect clusters with acceptor-like negatively charged traps in the $n^{0}$-layer, which arise as a result of electron emission from states located above the middle of the band gap. It was found that the differences in capacitance-voltage characteristics of the structures grown in hydrogen or argon ambient after irradiation are due to different doses of irradiation of GaAs $p^{+}-p^{0}-i-n^{0}$ structures and different degrees of compensation of shallow donor impurities by deep traps in the layers.
\end{abstract}

